\title{
In Silico Evaluation of Treatment of Periprosthetic Fractures in Elderly Patients After Hip Arthroplasty
}

\author{
Jacek Lorkowski, Renata Wilk, and Mieczyslaw Pokorski
}

\section{Abstract}

The aim of this study was to investigate the soundness of in silico finite element model (FEM) in the assessment of strain in the femur and in the components fixing the periprosthetic fracture in elderly patients after hip arthroplasty. From a group of 55 patients, aged 27-95, treated due to fractures after hip replacement in 2012-2018, 18 patients were separated out, aged over 85 , out of whom 7 had type $\mathrm{C}$ fractures, according to the Vancouver classification. These seven patients formed the study group. The fractures were stabilized with a locking compression plate system and wire loops or by replacement of the endoprosthesis stem. The FEM was performed by processing radiological images of the femur, considering the stabilization type and osteoporotic bone characteristics. Each patient's FEM was counter compared to virtual in silico control showing a non-osteoporotic

J. Lorkowski $(\bowtie)$

Department of Orthopedics and Traumatology, Central

Clinical Hospital of Ministry of Interior, Warsaw, Poland e-mail: jacek.lorkowski@gmail.com

R. Wilk

Department of Anatomy, Health Science Department, Medical University of Silesia, Katowice, Poland

Hope Medical Institute, Newport News, VA, USA

M. Pokorski

Institute of Health Sciences, Opole Medical School, Opole, Poland bone structure. We found that the strain was distinctly greater at the bone-implant interface after surgical stabilization with a multi-hole plate and cerclage wire loops in osteoporotic periprosthetic fractures when compared to the virtual non-osteoporotic bone. We conclude that the in silico model enables the assessment of strain distribution at the bone-implant interface, which helps identify the biomechanical incongruity of traditional bone stabilization methods in patients with osteoporotic bones.

\section{Keywords}

Elderly patients $\cdot$ Endoprosthesis $\cdot$ Finite element model · Hip arthroplasty · In silico · Periprosthetic fracture

\section{Introduction}

Hip arthroplasty is a frequent procedure to improve quality of life, extend lifespan, and maintain mobility in osteoarthritis (Marshall et al. 2017). In the USA, more than 300,000 such procedures are performed annually, mostly in the elderly people. Technological improvements in implant production and advances in endoprosthesis implantation cause that treatments are carried out in increasingly elderly patients with weak bone structure (Caruso et al. 2018). While the procedure of implant placement usually ends successfully, complications such as 
periprosthetic fractures are on the rise, reaching $1.7-4.1 \%$ of all arthroplasty procedures (Lee et al. 2018; Marshall et al. 2017). The fractures appear more often after revision arthroplasty than after primary endoprosthesis placement, $6 \%$ vs. $1 \%$, respectively. The most frequent cause of periprosthetic fracture is a fall on the flat surface caused by slipping or tripping. However, there also are fractures without previous injury, which points to bone weakness that raises concern in the elderly over 85 years of age who, additionally, may have various comorbidities (Chou and Davis 2017; Marsland and Mears 2012). Female gender, osteoporosis with bone mass loss, rheumatoid arthritis, and osteolytic lesions, particularly in bone areas exposed to load, predispose to fractures (Caruso et al. 2018). According to the Swedish National Hip Arthroplasty Registry, the incidence of periprosthetic fractures is second only to bone infections as complications after arthroplasty, raising socioeconomic issues due to morbidity and mortality (Karrholm et al. 2016). Several classification systems have been develop to assess periprosthetic fractures, which have a varying ability to characterize the fracture in radiographs, the condition of the tissue surrounding bone implant, and the implant fixation (Tower and Beals 1999; Cooke and Newman 1988; Johansson et al. 1981). Currently, Vancouver classification is commonly used. It enables the assessment of fracture size, prosthesis required, and the selection of the best treatment method (Duncan and Haddad 2014).

The Vancouver classification consists of the following facture types:

- Type A, fracture passing through the greater and lesser trochanters;

- Type B, fracture along the length of endoprosthesis, passing through the medial margin of the femur, which is further subdivided into:

- Type B1, with preserved implant stability and good quality of bone matrix around the implant;

- B2, with loose endoprosthesis but good quality of bone structure;
- B3, with loss of stability of endoprosthesis and bone of deficient structure;

- Type C, fracture extending below the endoprosthesis (Marshall et al. 2017).

The assessment of implant fixation in type B and $\mathrm{C}$ fractures is often subjectively made by a surgeon who performs anastomosis (Huang et al. 2018), which may have a bearing on a greater number of reoperations in these categories. Type $\mathrm{C}$ fractures create the largest clinical difficulty since they hamper balance and locomotor stability, often resulting in repeat fractures (Pavone et al. 2019; Randelli et al. 2018). The current standard is to schedule surgery, based on the evaluation of the patient's condition supported by radiographs. In advanced age, extensive procedures take toll on the patient, so that surgical decisions should be weight in with caution. In such situations, the finite element method (FEM), based on a rapid 2D modeling, seems of potential applicability, to assess the distribution of strain occurring in the implanted bone. Therefore, the main purpose of this study was to investigate the soundness of an in silico FEM model for the assessment of strain in the femur and in the components fixing the periprosthetic type $\mathrm{C}$ fractures in elderly patients after hip arthroplasty.

\section{Methods}

In this retrospective study, 18 elderly patients, aged over 85, were separated out of a group of 55 patients, aged 27-95, treated due to femur fractures after hip replacement surgery performed between 2012 and 2018. Seven out of the 18 patients had type $\mathrm{C}$ fractures, according to the Vancouver classification. These seven patients formed the study group. The fractures were stabilized with a locking compression plate system and wire loops or by replacement of the endoprosthesis stem. Out of the remaining 18 patients, 5 had type B1, 3 had type B2, and another 3 had type B3 fractures. Type B fractures, usually occurring in bones having better structural quality, were not considered in this study. 
Treatment outcome in type $\mathrm{C}$ fractures was evaluated 1 year after surgery using the Harris Hip Score (HHS) for the assessment of pain and joint mobility. The mean HHS score for this group was $71 \pm 4.4$ (SD) out of 100 points possible to achieve, which is considered a fair result (Harris 1969). In silico rapid modeling of fracture stabilization was based on patients' X-ray images of the femur obtained in the process of diagnosis and treatment. The method evaluates the strain arising in solid-state elements, which the bone belongs to, in the area initially divided into a specific number of simple finite elements that connect to each other at certain points in the periphery, called nodes (Lorkowski et al. 2018). After determining the distribution of the physical quantity inside finite elements, i.e., shape functions of nodes, calculations of differential equations for nodes are performed, while in the remaining parts of finite elements, the equation solutions are approximated. Then, boundary conditions are introduced into the system of equations, and the values of the physical quantities of interest in the nodes of interest are obtained. In this study, the degree and type of displacement in the nodes were determined, which allowed for the calculation of bone strain values.

Patients' X-ray images were used to make a geometric model of the femur with the implant and anastomosis used to stabilize the periprosthetic fracture. The model simulated fixation used for a fracture and stabilization depending on the bone parameters in elderly patients. Each osteoporotic patient's FEM was counter compared to virtual in silico control, containing the assumed healthy-like bone structure of a young adult with the corresponding distribution of elemental strains. The images were subjected to in silico modeling combining bone dynamics and FEM simulation, which allowed to visualize specific changes occurring in bones with different than normal characteristics, e.g., due to osteoporosis but also caused by the type fixation and by the endoprosthesis itself. The FEM model was created by automatically converting X-ray images considered as a bitmap composed of 256 grayscale elements in the computer program CT2FEM 1.0. The program assigns the appropriate FEM value to each modulus of the grayscale element. Next, the created model was loaded into Ansys Discovery Design software (Canonsburg, PA) to divide the finite elements into groups representing different tissues, such as compact or spongy bone, and the materials the endoprosthesis and fracture fixation components were made of.

Bone strain can be described by Young's coefficient and Poisson's coefficient for elasticity of solids. Young's modulus is the ratio of tension arising in the material to the elastic deformation caused under the influence of acting force. It varies depending on the tissue being analyzed. In our case, 4 values were important, representing the examined tissues and the metal used for fixation: $5 \mathrm{GPa}$ for compact bone, $3 \mathrm{GPa}$ for spongy bone, $300 \mathrm{MPa}$ for connective tissue and marrow cavity, and $210 \mathrm{GPa}$ for fixation metal. In case of advanced osteoporosis in the elderly, the above standard values representing Young's modulus of bone elements were halved. Poisson's coefficient, on the other side, is the ratio between transverse strain to the corresponding axial strain. It was determined as 0.32 for all the tissues. Fragments of the FEM model representing different regions could be turned off or on as separate subgroups for the analysis. All fragments of a given type of tissue were treated in the same way (Wirtz et al. 2000).

\section{$3 \quad$ Results}

Final analysis of in silico modeling included the following types of stabilization of type $\mathrm{C}$ fractures on osteoporotic patients:

1. Multi-hole plate, three cerclage wire loops, and four screws in the distal segment.

2. Multi-hole plate, three cerclage wire loops, and five screws in the distal segment.

The screws in the distal segment were passing through the whole bone, i.e., across the opposite layers of cortical bone. Additionally, at the level 
of endoprosthesis in the proximal segment, there were three screws, passing through only one cortical bone layer, and cerclage wire loops in both types of multi-hole plate stabilization above outlined. The results of FEM analysis obtained for either type of stabilization is exemplified below.

$\boldsymbol{R e} 1$ Peri-implant strain in an elderly patient with advanced osteoporosis and a femur fracture stabilized using a four-screw multi-hole plate and three cerclage wire loops was $792.215 \mathrm{MPa}$ at the fracture level (Fig. 1a). For comparison, the strain was reduced to $738.189 \mathrm{MPa}$, i.e., by $6.8 \%$ in the corresponding virtual model of a non-osteoporotic bone (Fig. 1b). Likewise, there were reductions in strain at the levels of the remaining three screws in the hypothetical young bone. Reductions in strain were also noticed both above and below the anastomosis. The largest strain in the patient's in silico model, double that present at the fracture site, appeared below the downmost screw, where there also was the largest strain reduction in the virtual model of young bone, exceeding $100 \mathrm{MPa}$.

$\boldsymbol{R e} 2$ Peri-implant strain in an elderly patient with advanced osteoporosis and a periprosthetic femur fracture stabilized with a five-screw multi-hole plate and three cerclage wire loops was $789.956 \mathrm{MPa}$ at the fracture level (Fig. 2a). The strain was reduced to $737.180 \mathrm{MPa}$, i.e., by about $7 \%$, in the corresponding virtual model of a control non-osteoporotic bone (Fig. 2b). Akin to the fourscrew stabilization model, the strain was increasing toward the distal bone segments in both true osteoporotic and virtual non-osteoporotic bone
Fig. 1 Frontal plane image. In silico finite element method (FEM) analysis of distribution of biomechanical strain in periprosthetic type $\mathrm{C}$ femur fracture stabilized with a multi-hole plate and four screws distally. Additional three shorter screws, passing through only one cortical bone layer, and three-wire couplings are visible in the proximal section: (a) real image of osteoporotic bone in an elderly patient, (b) virtual model corresponding to the same but non-osteoporotic, hypothetically healthy-like bone; red mark in "a" marks the fracture gap

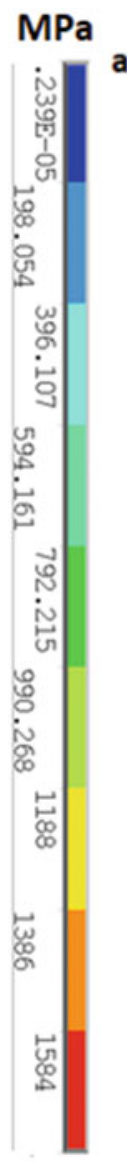

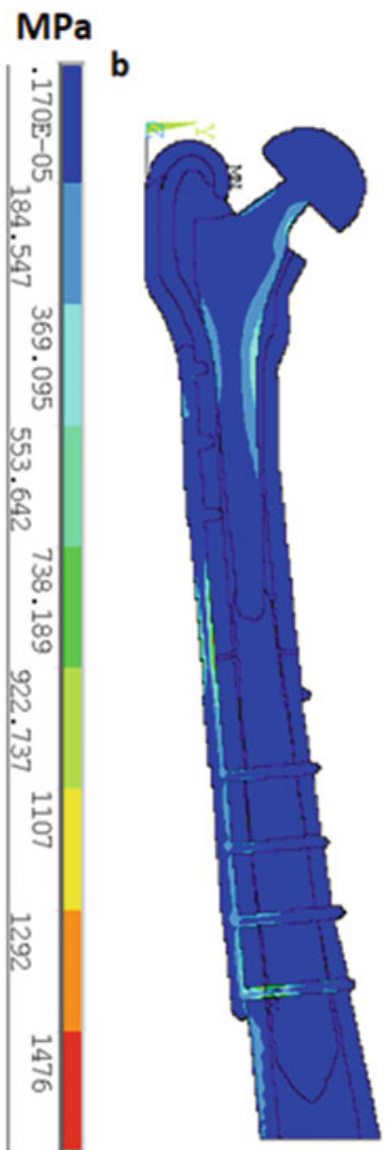


Fig. 2 Frontal plane image. In silico finite element method (FEM) analysis of distribution of biomechanical strain in periprosthetic type $\mathrm{C}$ femur fracture stabilized with a multi-hole plate and five screws distally. Additional three screws, passing through only one cortical bone layer, and three-wire couplings are visible in the proximal section: (a) real image of osteoporotic bone in an elderly patient, (b) virtual model corresponding to the same but non-osteoporotic, hypothetically healthy-like bone; red mark in "a" marks the fracture gap
$\mathrm{MPa}$

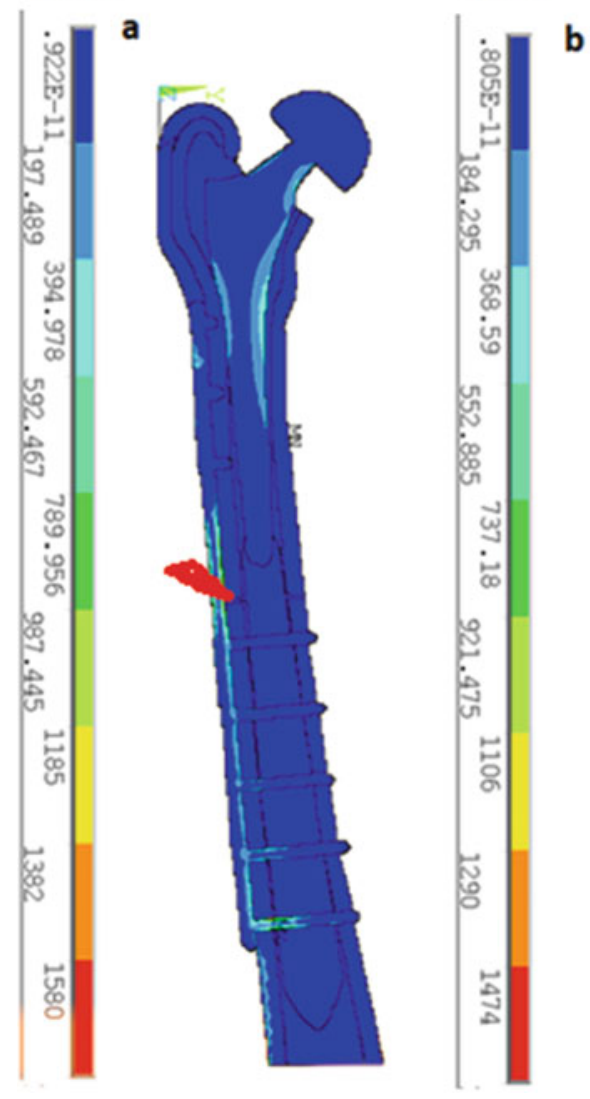

$\mathrm{MPa}$

b

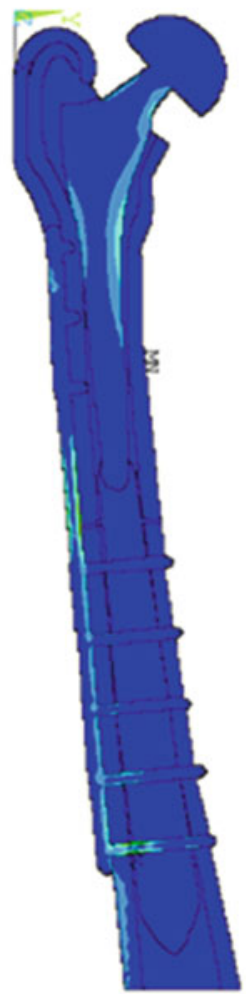

models, being correspondingly lower by about $10 \%$ in the latter bone model. The strain amounted to 1580.000 and $1474.000 \mathrm{MPa}$, i.e., by $6.7 \%$ in the downmost bone segment, respectively (Fig. 2b).

Strain evaluation was also made after stabilization of a prosthetic femur fracture using a multiplate four screws passing only through one cortical bone layer below endoprosthesis as seen in the proximal sections of the presented images. Differences between the true osteoporotic fractures and their corresponding virtual vis-avis were here less expressive. The bone strain was comparable in both osteoporotic and non-osteoporotic models above the fracture, in a range of $592 \mathrm{MPa}$ and $599 \mathrm{MPa}$. The maximum strain was noticed at the fracture level, amounting to 2,309 $\mathrm{MPa}$ in osteoporotic and 2,397 $\mathrm{MPa}$ in non-osteoporotic segments, an increase by about $4 \%$. Stabilization of a femur fracture using a multi-hole plate and five screws passing through one cortical layer provided comparable strain values (data not shown). Thus, mounting the plate only to the underlying cortical bone layer blurs the difference in strain between the osteoporotic and non-osteoporotic conditions and shows a fourfold increase in strain at the fracture level.

\section{Discussion}

Periprosthetic fractures are a serious complication in patients after hip arthroplasty. Especially, type $\mathrm{C}$ fractures that appear below the prosthesis shaft are challenging to treat. Typically, treatment includes dynamic compression plates, plates and 
cerclage wires, and non-locking plates. It is essential to choose the right length of a stabilizing plate. A shorter plate allows for a shorter surgical incision, reduces trauma to soft tissues, and shortens the surgery time. However, bone strain between the end of a prosthesis shaft and the proximal edge of a plate is high, risking a secondary fracture.

Another issue is the number of screws and wire loops used for the plate mount. Increasing their number and extent may cause unpredictable shifts in bone strain or raise substitute strains in other bone parts, leading to dysfunctional bone elasticity and eventually to prosthesis destabilization or repeat fractures (Lorkowski et al. 2014). Based on the present findings, we submit that mounting a metal plate using four screws, passing across the bone, with three cerclage wire loops suffices to achieve the optimum outcome. This conclusion stems from the results of in silico FEM modeling which showed that an increase in the number of screws to five caused a rather superfluous drop of about $4-5 \%$ in the strain along the femur bone, including the level of a multi-hole plate when compared to four screws. The assessment we made mostly concerned bone biomechanics. It did not include a biological context, for instance, peri-screw or bone itself infections or other bone pathologic conditions. It did include however osteoporosis, all too often occurring in elderly patients. Further, rapid comparing of the true patient's osteoporotic bone with its virtual non-osteoporotic, healthy-like counterpart enabled the assessment of changes in the strain distribution pattern caused by osteoporosis. We noticed that osteoporosis enhanced the bone strain around the multi-plate stabilator of a femur fracture by about $7 \%$, irrespective of the use of four or five screws. Nonetheless, the enhanced strain in periprosthetic fracture due to osteoporosis may be viewed as another argument to use fewer rather than more screws to maintain adequate osteoporotic bone elasticity.

When stabilizing a fracture, it is necessary to use cerclage wire loops above the prosthesis. Some surgeons also use additional shorter screws that pass through one cortical layer, reaching down to the level of an endoprosthesis (O'Toole et al. 2006). The in silico model of this study failed to confirm that the use of cerclage wire loops prevents the occurrence of a relatively high bone strain at the level of a multi-hole plate. Moreover, the use of plate screws passing through only one cortical layer at the level of endoprosthesis did not lead to a significant strain reduction along the entire length of bone subjected to anastomosis.

Choosing a correct method of stabilizing type $\mathrm{C}$ fractures is a matter of uncertainty in therapeutic decision-making. The questions arise to what extent plate osteosynthesis should be used and whether plate stabilization of a fracture should be combined with the revision prosthesis. An obvious disadvantage of stabilization with a plate, particularly in the elderly, is a longer time required for mobilization of a limb operated on. In case of revision arthroplasty, physical mobilization is possible much earlier. However, arthroplasty, particularly with additional plate stabilization, is a much more extensive and thus aggravating procedure. In principle, all types of anastomosis share similar doubts. In patients with periprosthetic fractures, treated in a classic manner with open reduction of bone displacement and internal fixation (ORIF), the percentage of failures reaches $33 \%$ in all fracture types (Pavone et al. 2019). Zuurmond et al. (2010) have shown that $12(55 \%)$ of 21 patients with type $\mathrm{C}$ fractures, who had a standard anastomosis with a non-locking plate or plates with cerclage wire loops, required reoperation. Better results have been noticed using locking plates in the less invasive stabilization system (LISS) for fracture fixation in another study, in which 11 of 12 patients have a post-surgery course free of complications (O'Toole et al. 2006). In the remaining patient, the plate broke down and required a replacement into a longer one. In another study, open reduction of bone displacement and double-plate fixation resulted in good outcome, with anastomosis union, in six of nine patients with type $\mathrm{C}$ periprosthetic femoral fractures (Lee et al. 2018). The three remaining patients had poor outcomes with nonunion, due mainly to 
osteoporosis. In two of those three patients (80 and 82 years of age), a secondary femur fracture took place below the anastomosis 2 and 4 weeks after fixation with double plates and wires. The two patients underwent revision surgery, using a double-plate anastomosis covering a longer length of a femur, with good long-term outcomes. In the third patient, in whom the plate failed to anastomose resulting in a new fracture, good outcome also was present 24 months after replacement for a double-plate anastomosis.

Patients with type $\mathrm{C}$ fractures supplied with classic ORIF stabilization have often recurrent fractures that are far more difficult to treat (Randelli et al. 2018). Another issue is revision prosthesis replacement, if required, using a compression plate to stabilize the periprosthetic fracture. We have previously described an elderly patient with perioperative fracture in whom the distribution of strain occurring during fracture stabilization was at the bone-anastomosis boundary greater compared to the average control. A high level of strain appeared linked to the excessive number of cerclage wire loops used (Lorkowski et al. 2014). These results give a consistent impression that in elderly patients with osteoporosis, treated with classic anastomosis, a check on the strain distribution over the bone should be an indispensable clinical intervention to achieve the best post-surgery outcome and to minimize a risk of secondary fractures and reoperations. To this end, FEM modeling enables the selection of the most appropriate method of anastomosis while scheduling surgery. A 2D version of FEM provides prompt results, approximately in 1-2 $\mathrm{h}$, which seems the optimum time to fix a fracture from the biomechanical standpoint, as opposed to $3 \mathrm{D}$ modeling that due to structural complexity requires 20 - to 30 -fold longer timeframe.

In patients with periprosthetic fractures, $\mathrm{X}$-rays are part of routine examination, as opposed to less used computed tomography. At present, X-rays are the best source for in silico FEM modeling. The method contains several software-driven simplifications that tend to unify various tissues examined. Therefore, a model contains approximations and rounding of details of bony structure, but the evaluation appears sufficient to illustrate changes taking place in the bone strain after application of different types of anastomosis. Further, the method appears wellsuited to appraise the level of osteoporosis, particularly in the elderly patient and spare additional complications and suffering related to surgical revisions, prosthesis exchanges, or repeat fractures.

FEM modeling, based on the solid-state biomechanics, was originally created to aid arthroplasty and orthopedic implants. Recently, the method has become of increasing interest due to potential diagnostic and therapeutic utility also in other medical fields, with some adaptations to fluid mechanics. The method has been studied, inter alia, for the prediction of an extent of post-traumatic lung injuries, which includes the evaluation of the accompanying lung inflammatory components (Danelson and Stitzel 2015; Lorkowski et al. 2015). Other novel applications of FEM extend to the evaluation of the biomechanical properties of the eustachian tube that connects the middle ear to the nasopharynx, which has to do with cartilage stiffness and mucosal adhesion in otitis media (Malik et al. 2016). Modeling of coiling deployment during endovascular interventions for intracranial aneurysms (Damiano et al. 2019) or the evaluation of a rupture risk of abdominal aortic aneurism (Siika et al. 2019; Soto et al. 2018) are other examples of this apparently burgeoning field of medicine. Biomechanical fingerprinting of lung tissue perfusion changes underlying inflammatory disorders seems an intriguing possibility to enhance the diagnostics of SARS-CoV2 pneumonitis and the like.

In conclusion, using the finite element modeling, we found that stabilization of type $\mathrm{C}$ periprosthetic fractures in the elderly patients is bound to enhance femoral strain at the boneimplant interface, due likely to osteoporotic bone changes. The strain is distinctly greater compared to that present in the young bone counterpart. In silico modeling enables the assessment of bone strain arising in periprosthetic fractures 
after hip arthroplasty, which helps identify the biomechanical incongruity of traditional bone stabilization methods.

Conflicts of Interest The authors declare that they have no conflicts of interest in relation to this article.

Ethical Approval All procedures performed in studies involving human participants were in accordance with the ethical standards of the institutional and/or national research committee and with the 1964 Helsinki Declaration and its later amendments or comparable ethical standards. Due to a retrospective nature of the material used in this study, a requirement to obtain approval from a local ethics committee as well as informed consent from individual participants were waived.

\section{References}

Caruso G, Milani L, Marko T, Lorusso V, Andreotti M, Massari L (2018) Surgical treatment of periprosthetic femoral fractures: a retrospective study with functional and radiological outcomes from 2010 to 2016. Eur J Orthop Surg Traumatol 28:931-938

Chou DTS, Davis B (2017) Trochanteric femoral fracture around a Birmingham hip resurfacing prosthesis: a case report and review of the literature. JBJS Case Connect 7(1):e7

Cooke PH, Newman JH (1988) Fractures of the femur in relation to cemented hip prostheses. J Bone Joint Surg Br 70(3):386-389

Damiano RJ, Tutino VM, Lamooki SR, Paliwal N, Dargush GF, Davies JM, Siddiqui AH, Meng $\mathrm{H}$ (2019) Improving accuracy for finite element modeling of endovascular coiling of intracranial aneurysm. PLoS One 14(12):e0226421

Danelson KA, Stitzel JD (2015) Finite element model prediction of pulmonary contusion in vehicle-to-vehicle simulations of real-world crashes. Traffic Inj Prev 16(6):627-636

Duncan CP, Haddad FS (2014) The unified classification system (UCS): improving our understanding of periprosthetic fractures. Bone Joint J 96(6):713-716

Harris WH (1969) Traumatic arthritis of the hip after dislocation and acetabular fractures: treatment by mold arthroplasty. An end-result study using a new method of result evaluation. J Bone Joint Surg Am 51:737-755

Huang JF, Jiang XJ, Shen JJ, Zhong Y, Tong PJ, Fan XH (2018) Modification of the unified classification system for periprosthetic femoral fractures after hip arthroplasty. J Orthop Sci 23(6):982-986

Johansson JE, McBroom R, Barrington TW, Hunter GA (1981) Fracture of the ipsilateral femur in patients with total hip replacement. J Bone Joint Surg Am 63 (9):1435-1442
Karrholm J, Lindahl K, Malchau H, Mohaddes M, Nemes S, Rogmark C, Rolfson O (2016) The Swedish hip arthroplasty register. Annual report 2016. https:// registercentrum.blob.core.windows.net/shpr/r/AnnualReport-2016-B1eWEH-mHM.pdf. Accessed on 12 Oct 2018

Lee J, Kim T, Kim T (2018) Treatment of periprosthetic femoral fractures following hip arthroplasty. Hip Pelvis $30(2): 78-85$

Lorkowski J, Mrzygłód MW, Kotela A, Kotela I (2014) Application of rapid computer modeling in the analysis of the stabilization method in intraoperative femoral bone shaft fracture during revision hip arthroplasty - a case report. Pol Orthop Traumatol 79:138-144

Lorkowski J, Mrzygłód M, Grzegorowska O (2015) Finite elements modeling in diagnostics of small closed pneumothorax. Adv Exp Med Biol 866:7-13

Lorkowski J, Grzegorowska O, Kozień MS, Kotela I (2018) Effects of breast and prostate cancer metastases on lumbar spine biomechanics: rapid in silico evaluation. Exp Med Biol 1096:31-39

Malik JE, Swarts JD, Ghadiali SN (2016) Multi-scale finite element modeling of Eustachian tube function: influence of mucosal adhesion. Int $\mathrm{J}$ Numer Method Biomed Eng 32(12):e02776

Marshall RA, Weaver MJ, Sodickson A, Khurana B (2017) Periprosthetic femoral fractures in the emergency department: what the orthopedic surgeon wants to know. Radiographics 37:1202-1217

Marsland D, Mears SC (2012) A review of periprosthetic femoral fractures associated with total hip arthroplasty. Geriatr Orthop Surg Rehabil 3(3):107-120

O'Toole RV, Gobezie R, Hwang R, Chandler AR, Smith RM, Estok DM, Vrahas MS (2006) Low complication rate of LISS for femur fractures adjacent to stable hip or knee arthroplasty. Clin Orthop Relat Res 450:203-210

Pavone V, de Cristo C, Di Stefano A, Costarella L, Testa G, Sessa G (2019) Periprosthetic femoral fractures after total hip arthroplasty: an algorithm of treatment. Injury 50(Suppl 2):S45-S51

Randelli F, Pace F, Priano D, Giai Via A, Randelli P (2018) Re-fractures after periprosthetic femoral fracture: a difficult to treat growing evidence. Injury 49 (Suppl 3):S43-S47

Siika A, Lindquist Liljeqvist M, Zommorodi S, Nilsson O, Andersson P, Gasser TC, Roy J, Hultgren R (2019) A large proportion of patients with small ruptured abdominal aortic aneurysms are women and have chronic obstructive pulmonary disease. PLoS One 14 (5): 0216558

Soto B, Vila L, Dilmé J, Escudero JR, Bellmunt S, Camacho M (2018) Finite element analysis in symptomatic and asymptomatic abdominal aortic aneurysms for aortic disease risk stratification. Int Angiol 37 (6):479-485

Tower SS, Beals RK (1999) Fractures of the femur after hip replacement: the Oregon experience. Orthop Clin North Am 30(2):235-247 
Wirtz DC, Schiffers N, Pandorf T, Radermacher K, Weichert D, Forst R (2000) Critical evaluation of known bone material properties to realize anisotropic FE-simulation of the proximal femur. J Biomech 33 (10):1325-1330
Zuurmond RG, van Wijhe W, van Raay JJ, Bulstra SK (2010) High incidence of complications and poor clinical outcome in the operative treatment of periprosthetic femoral fractures: an analysis of 71 cases. Injury 41 (6):725-733 